\title{
Rethinking clinical practice: clinical implementation of exome sequencing
}

\section{"Since the launch of the CLIA and CAP-accredited UCLA clinical exome sequencing test in January 2012, a number of patients have benefited from more precise and sometimes unpredictable diagnoses based on the test."}

\section{KEYWORDS: clinical exome sequencing $₫$ genetic diagnosis $\approx$ next-generation sequencing}

\section{Exome sequencing}

With the advent of next-generation sequencers and implementation [1] of capture technology [2], performing whole-exome sequencing has become routine, reliable and cost effective with assessment of approximately $95 \%$ of known protein-coding bases as well as splice sites. Since the first demonstration of successful identification of the disease-causing gene for Freeman-Sheldon syndrome by exome sequencing [3], many dozens of novel gene discoveries for rare Mendelian disorders have been empowered by it. Although improvements in both capture and sequencing technology as well as bioinformatics for variant calling are still highly valuable, we are comfortable about adapting this new technology to the clinical setting. There are already multiple groups offering clinical exome sequencing (CES) as a physician-ordered genetic test, and it is easily foreseeable that many additional groups will implement this powerful technology in the next few years. The College of American Pathologists has recently published a checklist specific to next-generation sequencing for clinical laboratory accreditation [101].

\section{Current medical practice}

Current medical practice for the diagnosis of rare diseases relies on detailed phenotyping of the patient prior to relatively focused genetic evaluation. In effect, the genetic evaluation is being used to confirm the clinical impression. Data are gathered by the physician using patient and family history as well as physical examination, which is highly cost effective. However, additional phenotyping including blood chemistry, imaging, biopsies, functional evaluation and so on are often expensive and frequently do not lead to a clear specific diagnosis. Longitudinal and repeat examinations are common and the diagnostic process can be frustrating to both the physician and patient. At the end of this diagnostic process, genetic testing is performed when indicated to confirm the diagnosis. Typically, this will be a single-gene test or a focused gene panel test for genetically heterogeneous disorders, followed by $6-12$ weeks of waiting for the result. However, with substantial variability of clinical presentations of human genetic disorders, coupled with the vast number of genes in the human genome, these genetic tests are often negative, leading to further serial genetic testing. There are currently approximately 3000 Mendelian disorders for which the genetic mutation is known and approximately 1700 gene tests offered globally [102]. Except for the few specific diseases with modest genetic heterogeneity such as Duchenne muscular dystrophy (DMD; MIM: 310200) and cystic fibrosis, the success rate of a genetic test or series of genetic tests is not very high. Accordingly, the ultimate costs to establish diagnoses of these rare diseases are high and patients do not benefit from a timely or precise genetic diagnosis.

\section{Examples of University of California, Los Angeles clinical cases where CES has provided a diagnosis \\ Since the launch of the Clinical Laboratory Improvement Amendments (CLIA) and College of American Pathologists-accredited University of California, Los Angeles (UCLA) CES test in January 2012 [103], a number of patients have benefited from more precise and sometimes unpredictable diagnoses based on the test. A few of those cases will be introduced here.}

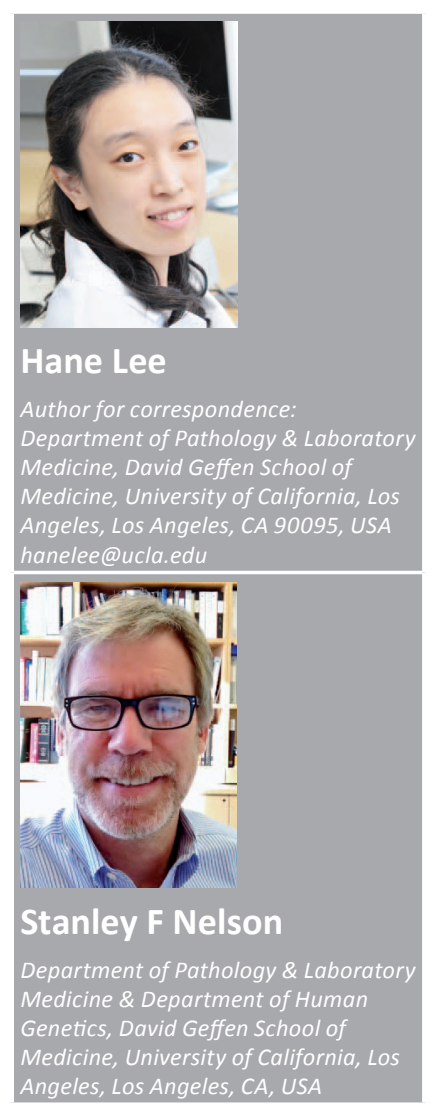

Future
Medicine $\mathrm{fS}$ 


\section{Autosomal dominant, de novo case: new diagnosis}

An approximately 12 -month-old male patient with gross motor developmental delay, mild dysmorphic features, hypertonia, consideration of a diagnosis of cerebral palsy and severe constipation with slow gastrointestinal motility was referred to the UCLA Genetics Clinic for CES of the unaffected parents and the patient after multiple pediatric subspecialty consults resulted in no firm diagnosis or clear path to diagnosis. CES of this trio identified a heterozygous de novo mutation in the canonical splice acceptor site of exon 9 of TCF4. Heterozygous truncating mutations are known causes of Pitt-Hopkins syndrome (MIM: 610954) [4]. This resulted in an early and clear diagnosis that ended the family's diagnostic odyssey much earlier than would have otherwise been possible. Notably, the child would not have met the diagnostic criteria that have been proposed to trigger the need for the sequence analysis of TCF4 mutations.

\section{Autosomal-recessive case: altered diagnosis}

An 18-year-old Persian female, clinically diagnosed with slowly progressive juvenile onset amyotrophic lateral sclerosis (ALS), was referred for CES in order to evaluate the known ALS genes for potentially causative mutations. Consistent with family history, sequence data confirmed evidence of a high degree of consanguinity. One heterozygous variant predicted to alter the function of the protein was identified within the known ALS gene, SETX [MIM: 602433], but could be ruled out as causal as $1.3 \%$ of the population carry the rare allele. From a search of all homozygous variants within the homozygous-by-descent areas of the genome, we identified a homozygous nonsense variant within the $A A A S$ gene. Recessive mutations in $A A A S$ cause achalasia-addisonianism-alacrima syndrome (triple-A syndrome/AAAS; MIM: 231550) [5]. A substantial fraction of the individuals with $A A A S$ gene mutations manifest neuropathy and spinobulbar amyotrophy. Upon identification of this mutation, which strongly indicated a diagnosis of AAAS, re-examination of the patient revealed that the patient had lifelong alacrima that had never been brought to medical attention. Furthermore, the child was operated on for achalasia 3 years prior to CES. The genetic finding from CES led to changing the diagnosis in this patient from juvenile ALS to AAAS, drastically altering the patient management and providing diagnostic clarity. This case stresses the significance and strength of the UCLA Clinical Genomic Board, where the knowledge of subspecialty physicians, geneticists, genetic counselors and bioinformaticians combine to retrieve and integrate all available information about the patient and correlate it with the genetic information in order to provide optimal diagnostic interpretation.

\section{DMD case with exonic duplication}

A 6-year-old male with proximal muscle weakness, positive Gower's sign, bilateral calf hypertrophy and an elevated CK level was referred for CES. No causal point mutations in any muscular dystrophy genes were detected genome-wide. With suspected DMD, exonic deletions and duplications mutation in $D M D$ were explored through normalized count ratios of patient compared with an aggregate set of unaffected male controls. An approximately 396-kb contiguous duplication of exons 3-20 was identified in DMD. Depth of coverage at a specific exon is highly consistent across samples in exome sequencing data and that is the basis of calling copy-number state [6]. Thus, determining copy-number variation with relative depth of coverage at each exonic region compared with the control samples provides sensitive detection of exonic deletions and duplications on chromosome $\mathrm{X}$ in males. Each individual exon within the duplication was 5 standard deviations away from the normal (nonmutant) sample mean.

\section{Challenges \& future perspective}

The accessibility and reliability of CES is not without challenges. In our experience, the success rate of CES leading to a clear diagnosis across a broad range of suspected Mendelian genetic diseases in this initial stage has been approximately $30 \%$. However, considering that most of the patients for whom the CES was ordered were those that had already exhausted all possible commercially available genetic tests, the 30\% success rate is sufficient to warrant broader-scale adoption.

\section{"This resulted in an early and clear diagnosis that ended the family's diagnostic odyssey much earlier than would have otherwise been possible."}

Two main challenges remain: interpretation challenges and technical challenges. The latter will be resolved with longer reads, improved capture approaches, or movement to whole-genome sequencing as reduced costs of sequencing make this economically reasonable. A subset of the $70 \%$ of patients with no significant mutation found 
probably harbor mutations that are undetectable by exome sequencing such as structural abnormalities, mutations in the regulatory regions that are not captured or expansion of tandem repeats. Identifying mutations in the regulatory regions will not only require sequencing those regions but also better informatics tools to predict which are pathogenic. Another subset of the unsolved patients will be due to the incorrect or incomplete phenotypic information provided by the physician. One of the strengths of UCLA CES test is that the patient's exome data are reviewed at a weekly genomic data board meeting consisting of an interdisciplinary team. It is not uncommon that the primary diagnosis is questioned and the phenotypes are viewed through a critical process that may result in changing the diagnosis completely. For outreach patients where the ordering physician cannot be physically present at the meeting, the physician is contacted for further phenotypic information or clarification of the chief complaint. With the vast amounts of data generated for each patient, this level of interactive interpretation process is important.

The last major subset of the unsolved patients will be those with mutations in novel genes that have not yet been associated with any human disorder. This will be a common scenario as there are still genes that remain undetected due to their rare frequency of mutation. However, with substantial effort to decipher the genetic basis of many Mendelian disorders in the research world, the pace of discovery is accelerating. Thus, it is important to note that patients evaluated one month and the next with the same genetic cause and sequence results could be given different interpretations. Patients should be informed that a 'negative' report is based on current knowledge and that periodic re-evaluation is warranted.

\section{"From our work and others, it is obvious that future medical practice will be changed drastically with more routine clinical exome sequencing and a shift to earlier genetic diagnosis."}

From our work and others, it is obvious that future medical practice will be changed drastically with more routine CES and a shift to earlier genetic diagnosis. When a patient presents with an unusual phenotype, the physician will take the family history and examine the patient. If one or more genetic causes are highly suspected as the proximate cause of the symptoms, CES will be ordered as a first-line test to establish the genetic diagnosis. Additional patient phenotyping will be guided by the genetic assessment. Also, most importantly and as quoted by one patient's mother in her interview with the Wall Street Journal [7], having a clear genetic diagnosis in hand means the lives of the patients and their families can be positively changed even without any treatment or therapy available at the time.

\section{Financial \& competing interests disclosure}

The authors have no relevant affiliations or financial involvement with any organization or entity with a financial interest in or financial conflict with the subject matter or materials discussed in the manuscript. This includes employment, consultancies, honoraria, stock ownership or options, expert testimony, grants or patents received or pending, or royalties.

No writing assistance was utilized in the production of this manuscript.

\section{References}

1 Metzker ML. Sequencing technologies - the next generation. Nat. Rev. Genet. 11(1), 31-46 (2010).

2 Lee H, O'Connor BD, Merriman B et al. Improving the efficiency of genomic loci capture using oligonucleotide arrays for high throughput resequencing. BMC Genomics 10 , 646 (2009).

$3 \mathrm{Ng} \mathrm{SB}$, Turner EH, Robertson PD et al. Targeted capture and massively parallel sequencing of 12 human exomes. Nature 461(7261), 272-276 (2009).

4 Whalen S, Heron D, Gaillon T et al. Novel comprehensive diagnostic strategy in Pitt-Hopkins syndrome: clinical score and further delineation of the TCF4 mutational spectrum. Hum. Mutat. 33(1), 64-72 (2012).
5 Handschug K, Sperling S, Yoon SJ, Hennig S, Clark AJ, Huebner A. Triple A syndrome is caused by mutations in AAAS, a new WD-repeat protein gene. Hum. Mol. Genet. 10(3), 283-290 (2001).

6 Sathirapongsasuti JF, Lee H, Horst BA et al. Exome sequencing-based copy-number variation and loss of heterozygosity detection: ExomeCNV. Bioinformatics 27(19), 2648-2654 (2011).

7 Marcus AD. Making gene mapping part of everyday care. Wall Street Journal, 14th May 2012.

\section{Websites}

101 Commission On Laboratory Accreditation. Laboratory Accreditation Program. Molecular Pathology Checklist. www.cap.org/apps/cap.portal? nfpb $=$ true $\&$ cntvwrPtlt_actionOverride $=\% 2 \mathrm{~F}$ portlets\%2FcontentViewer\%2Fshow\&_wind owLabel=cntvwrPtlt\&cntvwrPtlt\%7BactionF orm.contentReference $\% 7 \mathrm{D}=$ media resources\%2Fnewsrel_checklist_next_gene. html\&_state $=$ maximized $\&$ _ pageLabel $=$ cntvwr

102 GeneTests Medical Genetics Information Resource (database online). Copyright, University of Washington, Seattle, 1993-2012. www.genetests.org

103 Exome sequencing offers diagnostic clarity that can guide care decisions. www.uclahealth.org/body.cfm? xyzpdqabc $=0$ \&id=502\&action=detail \&ref=1057\&start=1 \&issueref $=178$ 